\section{Obesidade e maturação sexual precoce em escolares de Florianópolis - SC}

\section{Obesity and early sexual maturation among students from Florianopolis $-S C$}

\section{Fernando Adami ${ }^{1,2}$}

\section{Francisco de Assis Guedes de Vasconcelos²}

${ }^{1}$ Faculdade de Saúde Pública, Universidade do Estado de São Paulo, São Paulo, Brasil

1,2Programa de Pós Graduação em Nutrição da Universidade Federal de Santa Catarina (UFSC). Florianópolis, SC, Brasil

Projeto de Pesquisa financiado pelo Conselho Nacional de Desenvolvimento Científico e Tecnoló gico (CNPq), Processo no. 402322/2005-3.

Correspondência: Fernando Adami, Programa de Pós Graduação em Saúde Pública, Faculdade de Saúde Pública, Universidade de São Paulo. Avenida Dr. Arnaldo, 715, São Paulo, SP, CEP: 01246-904.

E-mail: adamifernando@uol.com.br.

\section{Resumo}

Objetivos: Investigar a associação entre maturação sexual precoce e obesidade em meninos e meninas de 10 a 14 anos de Florianópolis. Métodos: Participaram do estudo 629 escolares entre 10 e 14 anos (277 meninos e 352 meninas), obtidos de duas escolas públicas e duas escolas privadas da região central do município. Foi utilizado o índice de massa corporal (IMC) para determinação de sobrepeso e obesidade. Os valores de Z escore para IMC, peso e estatura foram determinados de acordo com sexo e idade. Para obtenção dos valores de Z escore, utilizou-se o método LMS. A maturação sexual foi avaliada de acordo com planilha contendo 5 estágios de maturação sexual para cada sexo. Os indivíduos foram agrupados por tercis da idade de acordo com estágio e sexo. $\mathrm{O} 1^{\circ}$ tercil foi considerado como maturação sexual precoce; e o $2^{\circ}$ tercil, como grupo de referência. Resultados: As meninas com maturação sexual precoce têm maiores valores de $\mathrm{Z}$ escore Peso $(\beta=-0,21, p<0,05)$, Z escore estatura $(\beta=-0,15$, $\mathrm{p}<0,05)$ e Z escore IMC $(\beta=-0,3, p<0,05)$ do que as meninas dos outros grupos. Já os meninos com maturação sexual precoce apresentam maiores valores de $\mathrm{Z}$ escore estatura ( $\beta=-0,21, p<0,05)$. As meninas com maturação sexual precoce têm duas vezes mais chances de ter sobrepeso, incluindo obesidade, do que meninas do grupo de referência, com associação significante $(p=0,01)$ Nos meninos, não foi encontrada associação significante $(p=0,648)$. Conclusão: Os achados deste estudo corroboram aqueles da literatura que afirmam que meninas com maturação sexual precoce têm maiores prevalências de sobrepeso, incluindo obesidade, do que as meninas do grupo de referência. Em relação aos meninos, o presente estudo não encontrou associação entre maturação sexual precoce e obesidade.

Palavras-chave: Obesidade. Maturação sexual. Escolares. Adolescentes. 


\section{Abstract}

Objective: To investigate the relationship between early sexual maturation and obesity among students aged 10-14 years from the city of Florianopolis. Methods: A total of 629 students (277 males and 352 females) aged 10-14 years were included in the study. The sample was obtained from two private schools and two public schools in the city of Florianopolis. The body mass index (BMI) was used for determining overweight and obesity. The values of weight, height, and BMI Z score according to gender and age were determined. The LMS method was utilized to determine $\mathrm{Z}$ score values. Sexual maturation was evaluated by Tanner stages for both males and females. Subjects were grouped using age-adjusted tertiles for each Tanner stage and gender. Subjects in the first tertile were considered early maturing, while subjects in the second tertile were considered the reference group. Results: Early maturing girls were heavier $(\beta=-0.21$, $p<0.05)$, taller $(\beta=-0.15, \mathrm{p}<0.05)$ and had higher BMI values for age $(\beta=-0.3, p<0.05)$ than girls of other groups. Early maturing boys were taller for age $(\beta=-0.21, p<0.05)$ than boys of other groups. The prevalence of overweight including obesity of early maturing girls was higher than for the reference group ( $p=0.01)$. No association was found between overweight, including obesity, and early sexual maturation among boys ( $p=0.648$ ). Conclusions: The results of this study are similar to other studies that declare that early maturing girls present a higher prevalence of obesity than non-early maturing girls. The present study did not show any association for boys regarding early sexual maturation and obesity.

Keywords: Obesity. Sexual Maturation. Students. Adolescents.

\section{Introdução}

A obesidade é considerada um dos maiores problemas de saúde pública da contemporaneidade ${ }^{1}$. Ela é definida como excesso de gordura corporal que está associado a quadros patológicos e de mortalida$\mathrm{de}^{2}$. Sua rede causal envolve a inter-relação entre aspectos genéticos e ambientais que influenciam a fisiologia e o comportamento do indivíduo. Como conseqüência, há um desequilíbrio no balanço energético capaz de produzir um armazenamento do excesso energético na forma de gordura ${ }^{3}$.

Para indivíduos adultos, a literatura aponta que a obesidade é fator de risco para mortalidade e diversos quadros patológicos, como doenças cardiovasculares, diabetes tipo II, certos tipos de câncer, doença da vesícula biliar, além de complicações de ordem psicológica ${ }^{4}$. Já em crianças e adolescentes, estudos também têm apontado que a obesidade é fator de risco para mortalidade, doenças cardiovasculares, diabetes tipo II, síndrome metabólica, entre outros quadros de morbidade ${ }^{5,6}$.

O quadro é agravado quando se analisam os dados de prevalência. No mundo, a obesidade nas duas primeiras décadas de vida tem aumentado numa taxa alarmante, nunca antes vista. Estudos têm apontado um incremento de três vezes em todo o mundo, sendo que esse aumento está acometendo tanto países desenvolvidos como países em desenvolvimento ${ }^{7}$. No Brasil, o incremento da obesidade infantil tem atingido taxas de crescimento comparáveis às dos Estados Unidos da América ${ }^{8}$.

A puberdade parece ser um período crucial para o desenvolvimento da obesidade $^{9,10}$. Nesse período é que acontece a maior diferenciação sexual desde a vida fetal e a mais rápida taxa de crescimento linear desde os primeiros anos de vida, além do ganho de estatura e peso que irão definir tais variáveis na idade adulta ${ }^{11}$.

O início adiantado e a maior velocidade de progressão da puberdade têm sido objeto de diversos estudos, cujo tema envolve a questão da puberdade e da obesidade. Tais 
fenômenos são determinados por meio da identificação de adiantamentos dos eventos da maturação sexual. A literatura tem amplamente usado os estágios de desenvolvimento sexual e a idade da menarca para identificar precocidade durante a puberdade. Assim, os indivíduos que têm o início da puberdade com idades mais tenras, bem como aqueles que atingem os estágios de maturação sexual também mais cedo, são considerados com maturação sexual precoce ${ }^{12}$.

Em meninas, dados têm apontado que a maturação sexual precoce está associada a maiores prevalências de sobrepeso e obesidade $^{13,14}$. Em meninos, poucos estudos têm sido feitos para averiguar a relação entre maturação sexual precoce e obesidade, e os resultados são ainda divergentes ${ }^{14,15}$.

Diante disso, investigar a influência da maturação sexual precoce na prevalência de obesidade pode auxiliar na adoção de medidas eficazes de prevenção da doença durante esse período do ciclo de vida. No Brasil não foram localizados estudos realizados com esse objetivo.

De acordo com investigação recente, Florianópolis é uma das capitais do Brasil que apresenta níveis acentuados de sobrepeso e obesidade na infância quando comparados com aqueles observados em outros municípios do país e em outros países ${ }^{16}$, o que enfatiza ainda mais a importância de se determinar períodos críticos e possíveis fatores de risco para a obesidade. No município, a prevalência de sobrepeso e obesidade tem sido examinada nas idades de 7 a 10 anos $^{17}$ e de 15 a 18 anos $^{18}$. Por meio do índice de Massa Corporal (IMC), os valores de sobrepeso, incluindo obesidade, detectados nestas investigações foram de 22,1\% e 11,4\%, respectivamente. No município, não foram localizados estudos realizados com o intuito de se identificar variáveis associadas à obesidade durante o período de puberdade.

Diante do exposto, o objetivo do presente artigo é analisar a associação entre maturação sexual precoce e obesidade em escolares de 10 a 14 anos de ambos os sexos do município de Florianópolis, SC.

\section{Métodos}

Este estudo é um subprojeto de uma pesquisa cujo objetivo principal é analisar a evolução da composição corporal, tendência e prevalência de sobrepeso, obesidade e baixo peso e sua relação com o estilo de vida em escolares de 7 a 14 anos de idade do município de Florianópolis, SC, nos anos de 2002 e 2007. A pesquisa foi aprovada pelo Comitê de Ética em Pesquisa com Seres Humanos da Universidade Federal de Santa Catarina, projeto $n^{\circ} 028 / 06$. O presente estudo é caracterizado como analítico transversal ${ }^{19}$.

A coleta de dados aconteceu entre os meses de Março e Abril de 2007, na cidade de Florianópolis, localizada no estado de Santa Catarina, região Sul do Brasil. De acordo com dados do IBGE, a população para 2007 que se encontra na faixa etária de 10 a 14 anos foi estimada em 35.817 habitantes $^{20}$. Segundo dados do Instituto Nacional de Estudos e Pesquisas Educacionais Anísio Teixeira (INEP), foram feitas, em 2006, 53.954 matrículas no ensino fundamental, sendo que de $5^{\mathrm{a}}$ a $8^{\mathrm{a}}$ série foram feitas 26.648 matrículas ${ }^{21}$.

Para o cálculo da amostra, foram considerados nível de significância de 95\% e poder de $80 \%$. A prevalência de indivíduos com maturação sexual precoce foi estabelecida em $25 \%$, com razão de chances em relação ao desfecho (obesidade) de $2 \%{ }^{15}$. O tamanho de amostra estimado para atender os critérios acima foi de 620 . Ainda foi acrescentado um valor de $10 \%$ para possíveis perdas na amostra. No total foram coletados dados de 651 escolares de 10 a 14 anos. Desses, foram válidos dados de 629 escolares (menos de $4 \%$ de perdas). $O$ cálculo foi feito no software epi-info.

Para a seleção da amostra, foram selecionadas quatro escolas, sendo duas públicas e duas privadas, que se encontram na região central de Florianópolis, onde há concentração de quase $50 \%$ de todos os alunos matriculados do município. Os alunos foram estratificados segundo sexo e idade em cada escola. A escolha dos alunos 
foi feita de forma aleatória. Os critérios de inclusão foram: ter idade entre 10 e 14 anos, ter consentimento informado autorizado pelos pais ou responsáveis e querer participar estudo.

Foram realizados dois workshops para treinamento da equipe de coleta de dados em relação aos procedimentos adotados. Posteriormente foi realizado um estudo piloto numa escola diferente das amostradas.

A coleta das medidas de peso e estatura seguiram normas internacionais de padronização ${ }^{22}$. Para a medida de peso, o indivíduo permanecia na posição ortostática (de pé, com o corpo ereto), com o peso dividido em ambos os pés, mantendo a cabeça de acordo com o plano de Frankfurt, ombros descontraídos e braços soltos lateralmente. O avaliado estava descalço e usando roupas leves (de preferência sem calça jeans, moletons, jaquetas). Para a medida de estatura, o avaliado permanecia também na posição ortostática, pés juntos, peso dividido em ambos os pés, parte superior das costas tocando o estadiômetro, cabeça de acordo com o plano de Frankfurt, ombros descontraídos e braços soltos lateralmente. Além disso, o avaliado deveria respirar fundo e manter a respiração. Esta técnica tem como objetivo diminuir o efeito da compressão gravitacional diária.

A forma de avaliação da maturação sexual foi por meio de auto-avaliação. $O$ procedimento adotado foi o seguinte: após a coleta de peso e estatura, os escolares se dirigiam a uma outra sala, onde aconteceria a avaliação da maturação sexual. Só nessa sala é que os avaliados tiveram acesso às figuras respectivas aos estágios de maturação sexual. Havia um pesquisador experiente na sala que dava explicações sobre como o escolar deveria proceder. No momento da escolha do estágio, o avaliado ficava sozinho na sala.

Para o comparativo de peso, estatura e Índice de Massa Corporal (IMC - kg/ $\mathrm{m}^{2}$ ) através das diferentes idades, foram utilizados os valores de $Z$ escore de peso ( $Z$ escore Peso), estatura ( $Z$ escore Estatura) e
IMC ( $\mathrm{Z}$ escore IMC) por idade. Para o cálculo desses valores de $\mathrm{Z}$ escore, utilizou-se os valores de LMS de acordo com a idade e o sexo. O método LMS resume os dados em três curvas suavizadas, específicas para cada extrato, no caso aqui as idades. O parâmetro $\mathrm{M}$ é o valor mediano do índice observado no interior de cada estrato; o parâmetro S representa o coeficiente de variação de cada estrato; e o parâmetro L é o coeficiente (Box-Cox) empregado para a transformação matemática dos valores da variável em questão com o objetivo de obter distribuição normal em cada estrato. $\mathrm{O}$ cálculo dos valores de $\mathrm{Z}$ escore foi feito com base na seguinte equação: $\mathrm{Z}$ Escore = $\left[(\mathrm{X} / \mathrm{M})^{\mathrm{L}}-1\right] /(\mathrm{LS})$, onde $\mathrm{X}$ são os respectivos valores de peso, estatura ou IMC ${ }^{23,24}$.

Os valores de referência de LMS para peso e estatura foram obtidos do Center for Disease Control and Prevention (CDC) ${ }^{25}$. Os valores de LMS para IMC foram obtidos da referência brasileira ${ }^{23}$. Dessa forma, peso, estatura e IMC foram analisados como variáveis contínuas. O IMC também foi analisado como variável categórica (sobrepeso, obesidade e não sobrepeso). Os pontos de corte para determinação de sobrepeso e obesidade foram obtidos da referência brasileira ${ }^{23}$.

A maturação sexual foi avaliada segundo critérios propostos por Tanner ${ }^{12}$. Foram utilizados os estágios de desenvolvimento de órgão que consistem em 5 estágios para desenvolvimento de órgão genital para meninos e seios para meninas. $\mathrm{O}$ estágio 1 representa o estado infantil (pré-púbere) e o estágio 5 é indicador de estado maduro adulto. O estágio 2 indica o início do desenvolvimento maturacional. Os estágios 3 e 4 indicam continuidade no processo maturacional. $\mathrm{O}$ instrumento utilizado foram a planilha de desenvolvimento de seios nas meninas e a de órgão genital nos meninos, ambas recomendados pelo Ministério da Saúde ${ }^{26}$, que foram adaptados para o presente estudo com algumas modificações nas ilustrações: contornos dos órgãos genitais (principalmente seios) e contrastes de cores. 
Foi realizada uma análise de consistência interna nos dados de auto-avaliação da maturação sexual. No instrumento de referência utilizado constavam os cinco estágios de desenvolvimento sexual propostos por Tanner (1962), com a diferença de que os estágios estavam descritos, e não mais desenhados. O tamanho da amostra para a validação foi determinado em $10 \%$ do total da amostra investigada, sendo estratificada por sexo e idade. Foram coletados dados de apenas uma escola. No total foram avaliados 30 indivíduos (16 meninas e 14 meninos). As variáveis idade, peso, estatura, IMC e os valores de Z escore IMC, peso e estatura não foram diferentes em relação à amostra total (teste $\mathrm{t}, \mathrm{p}>0,05$ ). Não foram encontradas diferenças entre as duas medidas de maturação sexual para meninos e meninas (Teste de Wilcoxon, $p>0,05)$. O coeficiente de correlação de Kendall foi de 0,627 ( $\mathrm{p}<0,01)$ para meninos e $0,739(\mathrm{p}<0,01)$ para meninas.

A determinação da maturação sexual precoce foi feita com base em tercis da idade decimal para cada um dos 5 estágios de maturação sexual, em cada sexo. Assim, para cada estágio de maturação sexual, os indivíduos foram distribuídos segundo idade, tendo sido classificados da seguinte forma: indivíduos com maturação sexual precoce, aqueles que se encontrassem no $1^{\circ}$ tercil; indivíduos com maturação sexual tardia, aqueles que se encontrassem no $3^{\circ}$ tercil; e grupo de referência, aqueles que se encontrassem no $2^{\circ}$ tercil.

Foram feitos testes de distribuição normal para as variáveis investigadas. O teste de normalidade utilizado foi o de KolmogorovSmirnov. Posteriormente, foi realizada análise estatística descritiva das variáveis do estudo, com a determinação de valores de tendência central e de variabilidade. Em relação à estatística inferencial, foram utilizados testes paramétricos, tendo em vista que os dados apresentavam distribuição normal (Teste t, Anova One Way/Post hoc: Tukey). Foi também realizada uma regressão linear simples para verificar se havia tendência linear entre os valores de $\mathrm{Z}$ escore de peso, estatura e IMC e os tercis de idade para os estágios de maturação sexual. Foi feito o teste qui-quadrado $\left(\chi^{2}\right)$, e a regressão logística para verificar a associação entre a exposição (maturação sexual precoce) e o desfecho (sobrepeso incluindo obesidade). Da regressão logística foram obtidos os valores de razão de chances e intervalo de confiança de 95\% (95\% IC). As co-variáveis independentes foram idade e tipo de escola (pública e privada). O teste $\chi^{2}$ também foi utilizado para verificar associação entre sobrepeso, incluindo obesidade, com sexo e com os tercis de maturação sexual. Foi considerado significante quando $\mathrm{p} \leq 0,05$. O software estatístico utilizado foi o SPSS versão 15.0.

\section{Resultados}

O número de perdas na amostra foi baixo, num total de 22 indivíduos (menos de 4\%). Desses, 7 indivíduos foram excluídos da amostra por baixa plausibilidade biológica (IMC maior ou menor que 4 desvios padrão). Os outros 14 foram excluídos por motivos de dados incompletos. Isso pode ser considerado um indicativo de consistência interna dos dados.

O Gráfico 1 mostra valores de mediana da referência brasileira ${ }^{23}$ e para a amostra desse estudo. O gráfico foi construído com os valores de mediana do IMC para cada 6 meses. A curva para a referência brasileira foi alisada com polinômio de $4^{\circ}$ grau para o sexo masculino e de $8^{\circ}$ grau para o sexo feminino ${ }^{23}$. Para os valores de mediana da amostra do presente estudo, optou-se por não fazer o alisamento, haja vista a maior dispersão dos dados. Para tal amostra, a média do número de indivíduos em cada extrato foi de 28 para meninos e 35 para meninas, sendo os valores mínimos e máximos de 13 e 44 para meninos e 17 e 52 para meninas. É evidente que os meninos deste estudo apresentam valores maiores da mediana do IMC do que os da referência nacional. A média da diferença entre as duas amostras foi de $2,1 \mathrm{Kg} / \mathrm{m}^{2}$, com o valor mínimo de $1,3 \mathrm{Kg} / \mathrm{m}^{2}$ (idade de 12,5 anos) e máximo de $3,7 \mathrm{Kg} / \mathrm{m}^{2}$ (idade de 10 anos). 

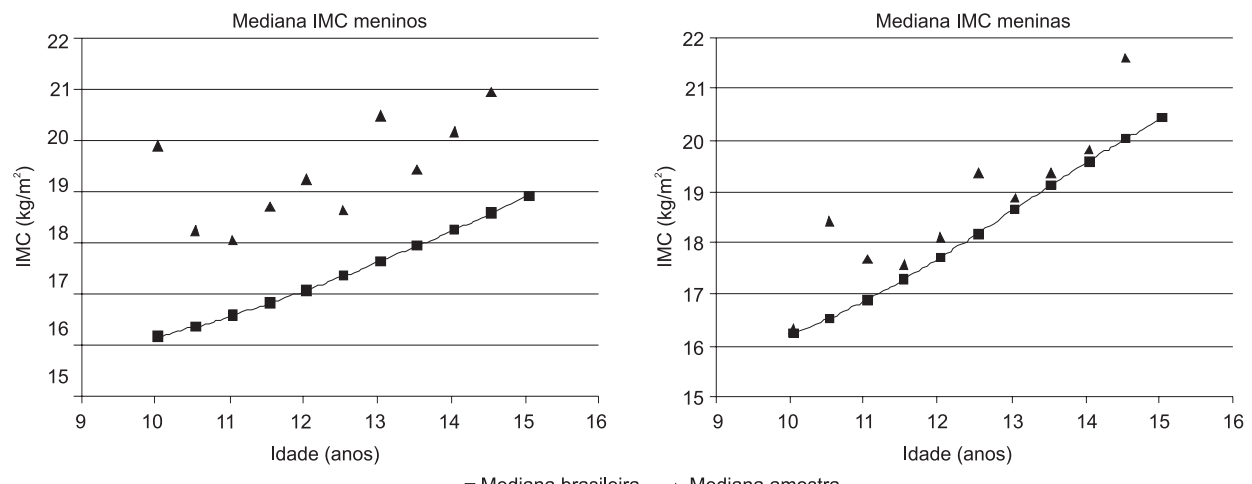

Gráfico 1 - Mediana do IMC para meninos e meninas da amostra comparada com a referência da população brasileira ${ }^{28}$, Florianópolis, SC, 2007.

Graph 1 - BMI median for the compared sample of boys and girls with reference values for Brazil28, Florianópolis, SC, 2007.

Já em relação às meninas, os valores de mediana do IMC são mais similares. A média da diferença entre as amostras foi de 0,7 $\mathrm{Kg} / \mathrm{m}^{2}$, com o valor mínimo de $0,1 \mathrm{Kg} / \mathrm{m}^{2}$ (10 anos) e o valor máximo de $1,9 \mathrm{Kg} / \mathrm{m}^{2}$ (10,5 anos). As maiores diferenças entre as meninas aconteceram nas idades de 10,5, 12,5, e 14,5. Cabe ressaltar, no entanto, que os dados da referência nacional são obtidos por meio de uma série de ajustes matemáticos, enquanto os dados da presente amostra são os próprios valores reais.
As características da amostra estão apresentadas na Tabela 1. Em relação às variáveis peso, $Z$ escore Peso, estatura, IMC eZ escore IMC, os meninos possuem valores maiores que as meninas. A prevalência de sobrepeso incluindo obesidade também é maior para os meninos. Meninos e meninas têm valores similares para a idade e $\mathrm{Z}$ escore Estatura. Portanto, a estatura por idade não é diferente entre os sexos.

A Tabela 2 apresenta valores das variáveis de acordo com a classificação de ma-

Tabela 1 - Média e desvio padrão (dp) de idade, variáveis antropométricas e prevalência de sobrepeso e obesidade para os escolares de 10 a 14 anos, Florianópolis, SC, 2007.

Table 1 - Mean and standard deviation for age, anthropometric variables and prevalence of overweight and obesity among students aged 10-14 years, Florianópolis, SC, 2007.

\begin{tabular}{lccc}
\hline Variáveis & Meninos $(\mathrm{n}=277)$ & Meninas $(\mathrm{n}=352)$ & $p$ \\
\hline Média $(\mathrm{dp})$ & Média $(\mathrm{dp})$ & 0,962 \\
\hline Idade (anos) & $12,8(1,3)$ & $12,8(1,2)$ & $0,002^{*}$ \\
Peso $(\mathrm{kg})$ & $50,8(13,1)$ & $47,8(11,2)$ & $0,004^{*}$ \\
Z escore Peso & $0,3(1,1)$ & $0,04(1)$ & $0,021^{*}$ \\
Estatura $(\mathrm{cm})$ & $157,9(11,6)$ & $156(9)$ & 0,265 \\
Z escore Estatura & $0,1(1,1)$ & $0,04(1)$ & $0,020^{*}$ \\
IMC (kg/m $\left.{ }^{2}\right)$ & $20,1(3,5)$ & $19,5(3,4)$ & $0,000^{*}$ \\
Z escore IMC & $0,8(1,2)$ & $0,1(1,1)$ & \\
& Prevalência (\%) & Prevalência (\%) & $0,001^{*}$ \\
Sobrepeso & 27,8 & 16,5 & \\
Obesidade & 5,8 & 5,1 & \\
\hline
\end{tabular}


turação sexual. O número de indivíduos em cada tercil foi acima de 90 para os meninos e acima de 115 para as meninas (ver a Tabela 2). Subdividindo cada estágio de maturação sexual em tercis de idade, o número médio de indivíduos foi de 18 para meninos e 23 para meninas. Os valores mínimo e máximo foram de 5 e 28 para meninos e 5 e 43 para meninas. Para ambos os sexos, os estágios 1 e 5 foram os que apresentaram menor número de indivíduos (n) por tercis de idade. No estagio 1 , para meninos o $\mathrm{n}$ foi de 5,6 e 6 para os tercis 1,2 e 3 , respectivamente. Nas meninas, esse $n$ foi de 5, 6 e 5 . No estágio 5 para os meninos, o $\mathrm{n}$ nesses tercis foi de 8,9 e 9 enquanto que para as meninas o $\mathrm{n}$ foi de 11 para cada tercil. Para ambos os sexos, o número de indivíduos em cada tercil foi similar. Quando comparados com os outros tercis, os dados demonstram que os meninos com maturação sexual precoce ( $1^{\circ}$ tercil) possuem valores similares de $\mathrm{Z}$ escore Peso, $Z$ escore IMC e de prevalência de sobrepeso, incluindo obesidade, e valores maiores de $\mathrm{Z}$ escore Estatura. A análise post hoc mostrou que os valores de $\mathrm{Z}$ escore
Estatura de meninos do $1^{\circ}$ tercil são maiores quando comparados com os valores de meninos do $2^{\circ}$ e do $3^{\circ}$ tercis. Também foi encontrada tendência linear negativa para os valores de $\mathrm{Z}$ escore Estatura em relação aos tercis de maturação sexual.

As meninas com maturação sexual precoce apresentaram maiores valores de $\mathrm{Z}$ escore Peso, Z escore Estatura e Z escore IMC. Houve também tendência linear negativa para os valores de tais variáveis em relação aos tercis de maturação sexual. A prevalência de sobrepeso, incluindo obesidade, de meninas com maturação sexual precoce também foi maior que a prevalência nos outros grupos. A análise post hoc mostrou que o $\mathrm{Z}$ escore Peso e o $\mathrm{Z}$ escore IMC das meninas com maturação sexual precoce foram maiores que os das meninas do $2^{\circ} \mathrm{e}$ do $3^{\circ}$ tercis. Em relação ao Z escore Estatura, a análise post hoc identificou diferenças entre o $1^{\circ}$ e o $3^{\circ}$ tercil.

Na Tabela 3, os valores de p e os de razão de chances para os meninos demonstram que não houve associação entre maturação sexual precoce e sobrepeso, incluindo obe-

Tabela 2 - Distribuição de idade, variáveis antropométricas e prevalência de sobrepeso e obesidade dos escolares de 10 a 14 anos segundo classificação de maturação sexual . Florianópolis, SC, 2007.

Table 2 - Distribution of age, anthropometric variables and prevalence of overweight and obesity among students aged 10-14 years according to sexual maturation status, Florianópolis, SC, 2007.

\begin{tabular}{|c|c|c|c|c|c|c|c|c|c|c|}
\hline \multirow{3}{*}{ Variáveis } & \multicolumn{5}{|c|}{ Meninos $(n=277)$} & \multicolumn{5}{|c|}{ Meninas $(n=352)$} \\
\hline & $\begin{array}{c}\text { Maturação } \\
\text { sexual } \\
\text { precoce } \\
(n=90)\end{array}$ & $\begin{array}{c}\text { Grupo de } \\
\text { referencia } \\
\quad(n=93)\end{array}$ & $\begin{array}{c}\text { Maturação } \\
\text { sexual } \\
\text { tardia } \\
(n=94)\end{array}$ & $\begin{array}{c}\text { Regressão } \\
\text { linear }(\beta)\end{array}$ & $p$ & $\begin{array}{c}\text { Maturação } \\
\text { sexual } \\
\text { precoce } \\
(n=115)\end{array}$ & $\begin{array}{l}\text { Grupo de } \\
\text { referencia } \\
(n=119)\end{array}$ & $\begin{array}{c}\text { Maturação } \\
\text { sexual tardia } \\
(n=118)\end{array}$ & $\begin{array}{c}\text { Regressão } \\
\text { linear }(\beta)\end{array}$ & $P$ \\
\hline & \multicolumn{5}{|c|}{ Média (dp) } & \multicolumn{5}{|c|}{ Média (dp) } \\
\hline Idade (anos) & $11,7(1)$ & $12,8(0,9)$ & $13,8(0,9)$ & - & $0,00^{*}$ & $11,7(1)$ & $12,8(0,9)$ & $13,9(0,8)$ & - & $0,00^{*}$ \\
\hline Peso (kg) & $47(11,9)$ & $49,7(12,2)$ & $55,5(13,8)$ & - & $0,00 *$ & $46,2(11,4)$ & $47,4(10,9)$ & $49,8(11)$ & - & $0,04^{*}$ \\
\hline Z escore Peso & $0,5(1,1)$ & $0,2(1)$ & $0,2(1,2)$ & $-0,11$ & 0,14 & $0,3(0,9)$ & $-0,01(0,9)$ & $-0,2(1)$ & $-0,21^{*}$ & $0,00^{*}$ \\
\hline Estatura $(\mathrm{cm})$ & $153,3(11,2)$ & $157,4(11,2)$ & $162,7(10,4)$ & - & $0,00^{*}$ & $152,5(8,8)$ & $156,2(9,3)$ & $159,2(7,6)$ & - & $0,00^{*}$ \\
\hline Z escore Estatura & $0,4(1,1)$ & $0,1(1)$ & $-0,1(1,1)$ & $-0,21^{*}$ & $0,00^{*}$ & $0,2(1)$ & $0(1)$ & $-0,1(1)$ & $-0,15^{*}$ & $0,01^{*}$ \\
\hline IMC $\left(\mathrm{kg} / \mathrm{m}^{2}\right)$ & $19,7(3,3)$ & $19,8(3,3)$ & $20,7(3,8)$ & - & 0,10 & $19,7(3,7)$ & $19,2(3,3)$ & $19,5(3,3)$ & - & 0,61 \\
\hline \multirow[t]{2}{*}{ Z escore IMC } & $0,9(1,2)$ & $0,7(1,1)$ & $0,7(1,3)$ & $-0,07$ & 0,34 & $0,6(1)$ & $0,03(1)$ & $-0,2(1,1)$ & $-0,3^{*}$ & $0,00^{*}$ \\
\hline & \multicolumn{5}{|c|}{ Prevalência (\%) } & \multicolumn{5}{|c|}{ Prevalência (\%) } \\
\hline Sobrepeso & 31,1 & 31,2 & 21,3 & - & 0,42 & 24,3 & 13,4 & 11,9 & - & $0,00^{*}$ \\
\hline Obesidade & 6,7 & 3,2 & 7,4 & $\underline{-}$ & & 8,7 & 4,2 & 2,5 & - & \\
\hline
\end{tabular}


Tabela 3 - Associação entre maturação sexual precoce e sobrepeso incluindo obesidade em escolares de 10 a 14 anos, Florianópolis, SC, 2007

Table 3 - Association between early sexual maturation and overweight including obesity among students aged 10-14 years, Florianopolis, SC, 2007

\begin{tabular}{|c|c|c|c|c|c|c|}
\hline \multirow[b]{2}{*}{ Tercis de MS } & \multicolumn{3}{|c|}{ Meninos $(n=277)$} & \multicolumn{3}{|c|}{ Meninas $(n=352)$} \\
\hline & $\begin{array}{l}\text { Razão de chances } \\
(95 \% \text { IC) }\end{array}$ & $\begin{array}{l}\text { Razão de chances } \\
\text { ajustada }(95 \% \text { IC })^{* *}\end{array}$ & $\mathrm{p}^{+}$ & $\begin{array}{c}\text { Razão de chances } \\
(95 \% \text { IC) }\end{array}$ & $\begin{array}{l}\text { Razão de chances } \\
\text { ajustada }(95 \% \text { IC)** }\end{array}$ & $p^{+}$ \\
\hline $2^{\circ}$ & 1 & 1 & & 1 & 1 & \\
\hline $1^{\circ}$ & $1,1(0,7-1,6)$ & $1,1(0,5-2,4)$ & 0,65 & $1,9(1,2-3)$ & $2,4(1-5,7)^{*}$ & $0,01^{*}$ \\
\hline $3^{\circ}$ & $1,2(0,8-1,8)$ & $0,7(0,3-1,5)$ & 0,43 & $1,2(0,7-2,2)$ & $2,6(0,8-8,3)$ & 0,31 \\
\hline
\end{tabular}

sidade. Já para as meninas, foi demonstrada associação. Dessa forma, as meninas com maturação sexual precoce ( $1^{\circ}$ tercil) têm aproximadamente duas vezes mais chances de apresentar sobrepeso, incluindo obesidade, do que meninas do grupo de referência ( $2^{\circ}$ tercil), mesmo quando ajustado por idade e tipo de escola (público ou privada).

\section{Discussão}

Este estudo teve como objetivo investigar a relação entre sobrepeso e obesidade com maturação sexual precoce em meninos e meninas de 10 a 14 anos de Florianópolis. Os resultados apontam para um risco aumentado de meninas com maturação sexual precoce terem maiores prevalências de sobrepeso, incluindo obesidade. Essa tendência não pôde ser encontrada nos meninos.

A amostra foi calculada com base nos critérios usados por Ribeiro et al. ${ }^{15}$, cuja metodologia de identificação de maturação sexual precoce foi idêntica à utilizada no presente estudo. Além disso, foram escolhidas as quatro maiores escolas da região central de Florianópolis, nas quais há quase $50 \%$ do total do número de matriculas realizadas no município. O número de perdas na amostra foi baixo (menos de 4\%). Mesmo assim, os dados do presente estudo podem não ser representativos do município. A amostragem não probabilística, a alta dispersão dos dados de IMC ao longo dos extratos de idade (ver o Gráfico 1) e a baixa perda de amostra são argumentos que vêm ao encontro da hipótese aventada da não representatividade dos dados.

O baixo número de pessoas (n) nos estágios 1 (17 para meninos e 16 para meninas) e 5 (26 para meninos e 33 para meninas) se deve à faixa etária da presente amostra, compreendida entre 10 e 14 anos. Parent et al. ${ }^{27}$ fizeram uma revisão dos estudos que determinaram a idade de início da puberdade, identificada por meio do estágio 2 de desenvolvimento de seios segundo critérios de Tanner (1963), em meninas de diversos países. O valor médio de idade no respectivo estágio foi de 10,5 anos. Nos meninos, dados tem apontado a idade de 10 anos para o início da puberdade (identificada por meio do estágio 2 de desenvolvimento do órgão genital ${ }^{28}$. Em relação ao estágio 5 , a literatura aponta que o desenvolvimento de seios e órgão genital nos meninos se estende até a idade de 15, 16 anos $^{12,28}$. Portanto, era esperado esse baixo número de pessoas nos estágios 1 e 5.

Diversos estudos transversais têm demonstrado a associação entre indicadores maturacionais e obesidade em meninas ${ }^{13-15}$. A razão de chances (IC 95\%) das meninas com maturação sexual precoce apresentarem maiores prevalências de sobrepeso tem sido de $3,6(1,7-7,3)^{13}, 1,6(1,1-2,4)^{14}$ e 2,1 $(1,1-4)^{15}$. Os achados do presente estudo 
corroboram com os achados da literatura. Apesar do uso de diferentes metodologias para a identificação da maturação sexual precoce, a associação dessa variável com obesidade em meninas está sempre presente nos estudos sobre o tema.

Em relação a estudos de coorte, Biro et al. ${ }^{29}$ estudaram a relação entre a idade da menarca e indicadores antropométricos (peso, estatura, IMC) em 616 meninas brancas e 539 negras. A taxa de maturação sexual foi definida como maturação precoce (meninas que atingiram a menarca com idade menor que o percentil 20), maturação tardia (meninas com idade da menarca $\geq$ percentil 80) e maturação com início médio (meninas com idade da menarca entre os percentis 20 e 80 ). A grande diferença desse estudo é o seu aspecto longitudinal, o qual possibilitou que os indivíduos fossem avaliados anualmente, nas idades de 9 a 19 anos. Ao longo dos anos de seguimento, a mediana do IMC foi sempre maior para meninas com maturação sexual precoce, quando comparada com a de meninas que tiveram maturação média e tardia.

Outros estudos com delineamento de coorte têm investigado a relação entre variáveis antropométricas (Exemplo: IMC ou adiposidade) na infância - taxa de maturação sexual-IMC ou adiposidade na idade adulta em meninas ${ }^{9,10,30,31}$. A discussão que tem sido feita nesses estudos é se realmente existe associação entre maturação sexual precoce e obesidade na idade adulta, já que os dados têm destacado que os valores de IMC ou adiposidade na infância são importantes variáveis de confusão no modelo. Assim, inferências apontam que a obesidade na idade adulta seria mais dependente de altos valores de IMC ou adiposidade na infância, quando comparada com a maturação sexual precoce $^{10,30,31}$.

Tais estudos, no entanto, analisaram as variáveis antropométricas nas idades médias de nove ${ }^{10}$ doze $^{31} \mathrm{e} \mathrm{quatorze}^{30}$ anos. Nessas idades, muitas meninas já iniciaram a sua maturação sexual. Uma das conseqüências da puberdade nas meninas é o aumento do percentual de gordura corporal
(\% de gordura $)^{11}$. Dessa forma, os valores das variáveis antropométricas nessas idades já podem refletir mudanças na composição corporal advindas da puberdade. Isso explicaria o resultado desses estudos que determinaram que a obesidade na idade adulta está relacionada à obesidade na infância, independente da taxa de maturação sexual.

Somente o estudo de Pierce \& Leon ${ }^{9}$ determinou que a maturação sexual precoce é um preditor de altos valores de IMC na idade adulta mais importante que o IMC na infância. O diferencial do estudo é a idade em que os pesquisados foram avaliados na infância: de 4 a 6 anos. Os autores ressaltam que, nessa idade, não há ainda influência da puberdade nas variáveis antropométricas. Os achados do estudo apontam para a relação entre maturação sexual precoce e obesidade na idade adulta. No entanto, há ainda a necessidade de mais estudos nessa área.

Dados sobre a relação entre maturação sexual precoce e obesidade no sexo masculino têm sido relatados. Os achados encontrados apontam para nenhuma relação entre as variáveis ${ }^{30}$, bem como uma relação de proteção ${ }^{14}$ e de risco ${ }^{15}$ de meninos com maturação sexual precoce terem maiores prevalências de sobrepeso e obesidade. Dados de estudos de coorte têm demonstrado que meninos com maturação precoce apresentam, na idade adulta, maiores valores de adiposidade (medidos por meio de dobras cutâneas) ${ }^{32}$, adiposidade central (medida por absortometria de raio $\mathrm{X}$ de dupla energia - DEXA) ${ }^{33}$ e circunferências da cintura e do quadril ${ }^{34}$. Van Lenthe et al. ${ }^{32}$, entretanto, observaram que nas idades de 13, 14 e 15 anos a adiposidade nos meninos com maturação sexual precoce diminuiu, enquanto que nos meninos com maturação sexual tardia ela aumentou.

Os dados do presente estudo apontam que meninos e meninas com maturação sexual precoce têm maior estatura para a mesma idade do que aqueles que não têm maturação sexual precoce. Karpati et al. ${ }^{35}$ analisaram dados de dois levantamentos 
realizados nos Estados Unidos: o National Health Examination Survey ciclos II e III (HES II e III), conduzido na década de 60, e o III National Health and Nutrition Examination Survey (NHANES III), conduzido entre os anos de 1988 e 1994. Os dados apontam que meninos do NHANES III apresentaram adiantamento da idade de início da puberdade, bem como maiores estaturas nas idades de 8 a 14 anos (diferença média de $2 \mathrm{~cm}$ ). Já Sanhu et al. ${ }^{34}$ encontraram que meninos que tiveram maturação precoce (identificada pelo $1^{\circ}$ quartil da idade da velocidade máxima de estatura) foram mais baixos na idade adulta quando comparados com cada um dos indivíduos dos outros quartis de idade da velocidade máxima de estatura. Nas meninas, Onland-Moret et al. ${ }^{36}$ realizaram uma revisão sistemática sobre idade da menarca e estatura adulta em países europeus. Os achados também apontam para a direção de que meninas de coortes mais atuais têm menor idade da menarca e maior estatura. No entanto, também se pode observar que meninas que tiveram maturação sexual precoce têm menor estatura na idade adulta do que meninas que não tiveram maturação sexual precoce.

A diferença dos achados longitudinais em relação aos achados transversais se deve provavelmente a questões de mudanças hormonais e crescimento físico. $\mathrm{He}$ \& Kalberg $^{37}$ encontraram que o ganho de IMC durante a infância estava associado a maior ganho de estatura durante a infância e ao adiantamento do início da puberdade. Portanto, é de se esperar que indivíduos que tenham maturação sexual precoce tenham maior estatura por idade durante os anos da puberdade. De fato, no estudo de Karpati et al. $^{35}$, a diferença nos valores de estatura ocorreu apenas nas idades de 8 a 14 anos, enquanto que em idades mais avançadas não houve diferenças na estatura.

Esse aumento de estatura devido à maturação sexual precoce pode estar associado ao aumento dos níveis de leptina que acontece durante a puberdade, tanto em meninos quanto em meninas ${ }^{38}$. Dados de estudos com culturas de células de tecido cartilaginoso demonstram que a administração de leptina nessas células estimula seu crescimento ${ }^{39}$, o que poderia estimular o crescimento da cartilagem epifisária encontrada nas extremidades dos ossos longos.

Entretanto, o início adiantado da puberdade tem como conseqüência a diminuição do período de crescimento pré-puberal devido ao fechamento dessa cartilagem epifisária dos ossos longos. O próprio estudo de He \& Kalberg ${ }^{37}$ encontrou que a maturação sexual precoce também estava associado a menores ganhos de estatura durante a adolescência. Dessa forma, mesmo que indivíduos com maturação sexual precoce possam ser mais altos do que os que não tem maturação sexual precoce, o menor ganho de estatura durante a adolescência sobrepõe o efeito benéfico de maior estatura durante os anos iniciais da puberdade dos indivíduos que têm maturação sexual precoce.

As meninas com maturação sexual precoce do presente estudo são, além de mais altas, mais pesadas, possuem maiores valores de IMC e maior prevalência de sobrepeso, incluindo obesidade, do que meninas que não tem maturação sexual precoce. O início da puberdade nas meninas está associado a um aumento na quantidade de massa gorda e massa magra ${ }^{38}$. Portanto, o adiantamento do início da puberdade pode ter como conseqüência o aumento de peso devido a mudanças teciduais. Além disso, com o aumento da concentração sanguínea de estradiol (um tipo de estrogênio), há maior estimulação de lipogênese em meninas com maturação sexual precoce ${ }^{11}$.

Para os próximos estudos sobre o tema, algumas questões devem ser analisadas pelos pesquisadores. O IMC na infância (antes dos 10 anos de idade) é uma variável de confusão importante a ser determinada num estudo de associação entre maturação sexual precoce $\mathrm{e}$ obesidade, tendo em vista os dados de estudos já apresentados aqui que apontam para tal variável como importante preditora dos valores de IMC na idade adulta. O próprio uso do IMC deve ser revisto para os próximos estudos. Apesar de o IMC ser recomendado para o diagnóstico do estado nutricional para pesquisas 
populacionais ${ }^{40}$, ele nãoécapaz de discriminar o tipo de tecido (muscular ou adiposo). Assim, indivíduos com o mesmo IMC podem ter diferentes percentuais de gordura corporal (\% de gordura). Com as mudanças corporais que acontecem ao longo das duas primeiras décadas de vida, essa não discriminação torna-se uma limitação muito importante ${ }^{41}$. Portanto, há a necessidade do uso de medidas mais acuradas de gordura corporal nos próximos estudos que investiguem a relação entre maturação sexual e obesidade.

Por fim, este estudo corrobora os achados na literatura de que meninas que apresentam maturação sexual precoce têm maiores prevalências de sobrepeso e obesidade. Em relação aos meninos, o estudo não conseguiu demonstrar associação.

\section{Referências}

1. World Health Organization. Diet, nutrition and the prevention of chronic diseases. WHO Technical report series 916. WHO/FAO, Geneva: 2002.

2. Haslam DW, James WPT. Obesity. Lancet. 2005; 366: 1197-209.

3. Speakman JR. Obesity: the integrated roles of environment and genetics. J Nutr 2004; 134: 2090-105S.

4. World Health Organization. Obesity: preventing and managing the global epidemic. WHO Technical report series 894. WHO, Geneva; 2000.

5. Thompson DR, Obarzanek E, Franko DL, Barton BA, Morrison J, Biro FM et al. Childhood overweight and cardiovascular disease risk factors: The National Heart, Lung, and Blood Institute Growth and Health Study. $J$ Pediatr 2007; 150: 18-25.

6. Engeland A, Bjorge T, Sogaard AJ, Tverdal A. Body mass index in adolescence in relation to total mortality: 32-year follow-up of 227.000 Norwegian boys and girls. Am J Med. 2003; 157: 517-23.

7. Ebbeling CB, Pawlak DB, Ludwig DS. Childhood obesity: public-health crisis, common sense cure. Lancet 2002; 360: 473-82.

8. Wang Y, Monteiro C, Popkin BM. Trends of obesity and underweight in older children and adolescence in the United Sates, Brazil, China, and Russia. Am J Clin Nutr. 2002; 75: 971-7.

9. Pierce MB, Leon DA Age at menarche and adult BMI in the Aberdeen Children of the 1950s cohort study. Am J Clin Nutr. 2005; 81: 733-9.

10. Freedman DS, Khan LK, Serdula MK, Dietz WH, Srinivasan SR, Berenson GS. The relation of menarcheal age to obesity in childhood and adulthood: the Bogalusa heart study. BMC Pediatr. 2003; 3(3): 1-9.

11. Rogol Ad, Roemmich Jn, Clark Pa. Growth at puberty. $J$ Adolesc Health. 2002; 32: 192-200.

12. Malina RM, Bouchard C. Growth, maturation, and physical activity. Champaign, Illinois: Human Kinetics Books; 1991.
13. Himes JH, Obarzanek E, Baranowski T, Wilson DM, Rochon J, Mcclanahan BS. Early sexual maturation, body composition, and obesity in African-American girls. Obes Res 2004; 12: 64-72S.

14. Wang Y. Is obesity associated with early sexual maturation? A comparison of the association in American boys versus girls. Pediatrics 2002; 110: 903-10.

15. Ribeiro J, Santos P, Duarte J, Mota J. Association between overweight and early sexual maturation in Portuguese boys and girls. Ann Hum Biol. 2006; 33: 55-63.

16. Assis MAA, Rolland-Cachera MF, Vasconcelos FAG, Bellisle F, Calvo MCM, Luna, MEP et al. Sobrepeso e baixo peso em crianças de 7 a 9 anos de idade de Florianópolis, Sul do Brasil: uma comparação com estudo francês usando protocolo similar. Revista de Nutrição 2006; 19: 299-308.

17. Assis MAA, Rolland-Cachera MF, Grosseman S, Vasconcelos FAG, Luna MEP, Barros Filho MV et al. Obesity, overweight and thinness in schoolchildren of the city of Florianópolis, Southern Brazil. Eur J Clin Nutr 2005; 59: 1015-21.

18. Farias Junior JC, Lopes AS. Prevalência de sobrepeso em adolescentes. Revista Brasileira de Ciência e Movimento. 2003; 11: 77-84.

19. Pereira MG. Epidemiologia: teoria e prática. Rio de Janeiro; Guanabara Koogan: 2002.

20. Instituto Brasileiro de Geografia e Estatística IBGE. População de 10 a 14 anos do município de Florianópolis. Disponível em http:// tabnet.datasus.gov. br/cgi/tabcgi.exe?ibge/cnv/popsc.def. [Acessado em 30 de setembro de 2007].

21. Instituto Brasileiro de Geografia e Estatística - IBGE. Matriculas no ensino fundamental. Disponível em http://www.ibge.gov.br/cidadesat/default.php. [Acessado em 30 de setembro de 2007].

22. Lohman TG, Roche AF, Martorell R. Anthropometric standardization reference manual. Illinois: Human Kinetics Books, 1988. 
23. Conde WL, Monteiro CA. Brazilian body mass index cut off points for evaluation of nutritional status from children and adolescents. J Pediatr. 2006; 82(4): 266-72.

24. Cole TJ, Bellizzi MC, Flegal KM, Dietz WH. Establishing a standard definition for child overweight and obesity worldwide: international survey. Br Med J. 2000; 320: 1-6.

25. National Center For Health Statistics. CDC growth charts: United States. 2002. (http://www.cdc.gov/ growthcharts/). Acessada em Dezembro de 2007.

26. Ministério da Saúde (Ms). Normas de atenção à saúde integral do adolescente. Brasília; 1993.

27. Parent AS, Teilmann G, Juul A, Skakkebaek NE, Toppari J. Bourguignon, J.P. The Timing of Normal Puberty and the Age Limits of Sexual Precocity: Variations around the World, Secular Trends, and Changes after Migration. Endocrine Reviews 2003; 24: 668-93.

28. Sun SS, Schubert CM, Chumlea WC, Roche AF, Kulin HE, Lee PA et al. National estimates of the timing of sexual maturation and racial differences among US children. Pediatrics 2002; 110: 911-9.

29. Biro FM, Mcmahon RP, Striegel-Moore R, Crawford $\mathrm{PB}$, Obarzanek E, Morrison JA et al. Impact of timing of pubertal maturation on growth in black and white female adolescents: The National Heart, Lung, and Blood Institute Growth and Health Study. J Pediatr 2001; 138: $636-43$.

30. Bratberg GH, Nilsen TIL, Holmen TL, Vatten LJ. Early sexual maturation, central adiposity and subsequent overweight in late adolescence. A four-year follow-up of 1605 adolescent Norwegian boys and girls: the Young HUNT study. BMC Public Health. 2007; 7: 54.

31. Must A, Naumova EN, Phillips SM, Blum, M, DawsonHughes B, Rand WM. Childhood Overweight and Maturational Timing in the Development of Adult Overweight and Fatness: The Newton Girls Study and Its Follow-up. Pediatrics 2005; 116: 620-7.

32. Van Lenthe FJ, Kemper HCG, Mechelen WV. Rapid maturation in adolescence results in greater obesity in adulthood: the Amsterdam growth and health study. Am J Clin Nutr 1996; 64: 18-24.
33. Kindblom JM, Lorentzon M, Norjavaara E, Lonn L, Brandberg J, Angelhed JE et al. Pubertal Timing Is an Independent Predictor of Central Adiposity in Young Adult Males: The Gothenburg Osteoporosis and Obesity Determinants Study. Diabetes 2006; 55: 3047-52.

34. Sandhu J, Shlomo YB, Cole TJ, Holly J, Smith GD The impact of childhood body mass index on timing of puberty, adult stature and obesity: a follow-up study based on adolescent anthropometry recorded at Christ's Hospital (1936-1964). Int J Obes 2006; 30: 14-22.

35. Karpati AM, Rubin CH, Kieszak SM, Marcus, M, Troiano, RP. Stature and Pubertal Stage Assessment in American Boys: The 1988-1994 Third National Health and Nutrition Examination Survey. J Adolesc Health 2002; 30: 205-212.

36. Onland-Moret NC, Peeters PHM, Van Gils CH, ClavelChapelon F, Key T, Tjønneland A, Trichopoulou A et al. Age at menarche in relation to adult height. Am J Epidemiol 2005; 162: 623-32

37. He Q, Karlberg J. BMI in childhood and its association with height gain, timing of puberty, and final height. Pediatr Res 2001; 49: 244-51.

38. Ahmed ML, Ong KKL, Morrell DJ, Cox L, Drayer N, Perry L et al. Longitudinal Study of Leptin Concentrations during Puberty: Sex Differences and Relationship to Changes in Body Composition. J Clin Endocrinol Metab 1999; 84: 899-905.

39. Styne DM. The regulation of pubertal growth. Horm Res 2003; 60(S1): 22-6.

40. Wells JCK, Fewtrell MS. Measuring body composition. Arch Dis Child 2006; 91: 612-7.

41. Hall DMB, Cole TJ. What use is the BMI? Arch Dis Child 2006; 91: 283-6.

Recebido em: 15/01/08

Versão final reapresentada em: 24/07/08 Aprovado em: 07/08/08 